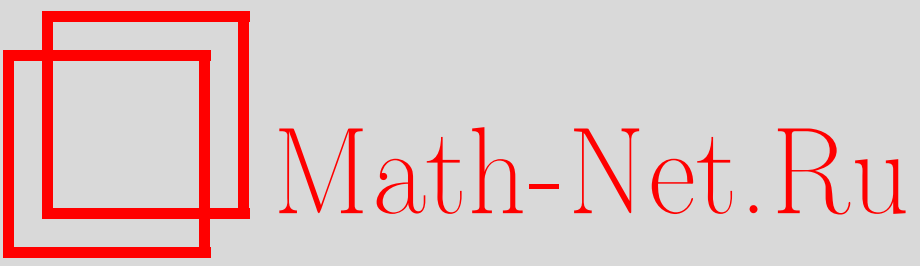

М. В. Павлов, Новые интегрируемые $(2+1)$-уравнения гидродинамического типа, УМH, 2003, том 58, выпуск 2, 171-172

DOI: https://doi.org/10.4213/rm621

Использование Общероссийского математического портала Math-Net.Ru подразумевает, что вы прочитали и согласны с пользовательским соглашением

http: //www. mathnet.ru/rus/agreement

Параметры загрузки:

IP : 3.85 .5 .30

26 апреля 2023 г., 17:05:56 


\title{
НОВЫЕ ИНТЕГРИРУЕМЫЕ $(2+1)$-УРАВНЕНИЯ ГИДРОДИНАМИЧЕСКОГО ТИПА
}

\author{
М. В. ПАВЛОВ
}

По аналогии с системами гидродинамического типа, интегрируемыми обобщенным методом годографа, [1],

$$
u_{t}^{i}=v_{j}^{i}(\mathbf{u}) u_{x}^{j}, \quad i, j=1,2, \ldots, N,
$$

введем понятие бесконечно-компонентных аналогов треугольного типа таких систем:

$$
\partial_{t^{n}} H_{k}=\partial_{x} F_{k, n}\left(H_{0}, H_{1}, \ldots, H_{k+n}\right), \quad k, n=0,1, \ldots, \quad F_{k, 0} \equiv H_{0}, \quad x \equiv t^{0} .
$$

Системы вида (2) в дальнейшем мы будем называть гидродинамическими цепочками.

ОПРЕДЕЛЕниЕ (см. [2]). Полугамильтонова (нераспадающаяся) система гидродинамического типа (1) назьвается егоровской, если она имеет пару законов сохранения вида

$$
\partial_{t} a(\mathbf{u})=\partial_{x} b(\mathbf{u}), \quad \partial_{t} b(\mathbf{u})=\partial_{x} c(\mathbf{u})
$$

Рассмотрим произвольное количество коммутирующих потоков

$$
u_{t^{k}}^{i}=w_{(k) j}^{i}(\mathbf{u}) u_{x}^{j}, \quad i, j=1,2, \ldots, N,
$$

где $t \equiv t^{1}$, и такое же количество плотностей законов сохранения $a_{n}(\mathbf{u})$. В этом случае законы

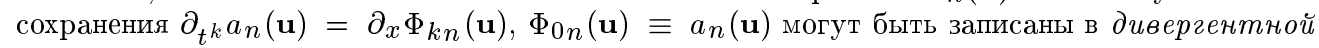
форме

$$
d \Omega_{i}=\Phi_{i k}(\mathbf{u}) d t^{k} .
$$

Лемма. Для любых коммутирующих потоков (4) найдутся такие плотности законов сохранения $a_{n}(\mathbf{u})$, что дивергентная форма (5) будет симметричной, и наоборот, для любых плотностей законов сохранения $a_{n}(\mathbf{u})$ найдутся такие коммутирующие потоки (4), что дивергентная форма (5) будет симметричной.

Всюду в дальнейшем будем рассматривать только егоровские гидродинамические цепочки, т.е. гидродинамические цепочки, имеющие пару законов сохранения вида (3). Потребуем, чтобы вторые производные от функции $\Omega\left(t^{0}, t^{1}, \ldots\right)$, зависящей (неявно) от бесконечного числа независимых переменных $t^{k}$, были (явнылми) функциями только от конечного числа полевых переменных (моментов) $H_{m}$, а именно, $\Omega_{n k}=\Phi_{n k}\left(H_{0}, H_{1}, \ldots, H_{n+k}\right)$, где $\Phi_{0 i} \equiv H_{i}, i=1,2, \ldots$. Тогда первые две егоровские гидродинамические цепочки из всей иерархии коммутирующих потоков имеют вид (здесь $y \equiv t^{2}$ )

$\partial_{t} H_{0}=\partial_{x} H_{1}, \quad \partial_{t} H_{1}=\partial_{x} \Phi_{11}\left(H_{0}, H_{1}, H_{2}\right), \quad \partial_{t} H_{2}=\partial_{x} \Phi_{12}\left(H_{0}, H_{1}, H_{2}, H_{3}\right), \quad \ldots$, $\partial_{y} H_{0}=\partial_{x} H_{2}, \partial_{y} H_{1}=\partial_{x} \Phi_{12}\left(H_{0}, H_{1}, H_{2}, H_{3}\right), \partial_{y} H_{2}=\partial_{x} \Phi_{22}\left(H_{0}, H_{1}, H_{2}, H_{3}, H_{4}\right), \ldots$

ТЕОРема. Первый коэффициент $\Phi_{11}\left(H_{0}, H_{1}, H_{2}\right)$ полностью определяет не только все остальные коэффичиенты дивергентной формы (5), но и соответств ующие интегрируемые $(2+1)$-мерные уравнения гидродинамического типа.

На промежуточном этапе вычислений (проверка равенства вторых смешанных производных $\left.\partial_{y} \Phi_{11}=\partial_{t} \Phi_{12}\right)$ возникает условие

$$
\frac{\partial \Phi_{11}}{\partial H_{2}}=\frac{1}{\alpha H_{0}+\beta H_{1}+\gamma+\varepsilon H_{2}}
$$

где $(\alpha, \beta, \gamma, \varepsilon)$-произволшные константы. Представляет интерес рассмотреть следующие случаи. 
1. Если положить $\alpha=\beta=\varepsilon=0(\gamma=1)$, то

$$
\Phi_{11}=H_{2}+\frac{A}{4} H_{1}^{2}+B H_{0} H_{1}+\frac{B^{2}}{A} H_{0}^{2}+C H_{1}+E H_{0}+G e^{-A H_{0}}
$$

где $(A, B, C, E, G)$ - произволшные константы. Вспоминая, что $H_{0} \equiv \Omega_{x x}, H_{1} \equiv \Omega_{x t}, H_{2} \equiv$ $\Omega_{x y}, \Phi_{11} \equiv \Omega_{t t}$, получаем новое интегрируемое $(2+1)$-мерное бездисперсионное уравнение

$$
\Omega_{t t}=\Omega_{x y}+\frac{A}{4} \Omega_{x t}^{2}+B \Omega_{x x} \Omega_{x t}+\frac{B^{2}}{A} \Omega_{x x}^{2}+C \Omega_{x t}+E \Omega_{x x}+G e^{-A \Omega_{x x}} .
$$

Если $C=E=G=0, A=-2 B^{2}$ и $B \rightarrow 0$, получаем хорошо известное уравнение Хохлова-Заболоцкой; если положить $G=-2 B^{2} A^{-3}+2 G_{0} A^{-2}$ и $A \rightarrow 0$, то получаем модифицированное уравнение Хохлова-Заболоцкой

$$
\Omega_{t t}=\Omega_{x y}+B \Omega_{x x} \Omega_{x t}+G_{0} \Omega_{x x}^{2}+\frac{B^{2}}{3} \Omega_{x x}^{3}
$$

в общем же случае с помощью галилеев ского преобразования выражение (7) приводится к более компактному виду

$$
\Phi_{11}=H_{2}+\frac{A}{4} H_{1}^{2}+G e^{-A H_{0}},
$$

что определяет нетривиальное обобщение (т.е. когда $G \neq 0$ ) иерархии бездисперсионного предела дискретного аналога уравнения Кадомцева-Петвиашвили (см. [3]). Таким образом, даже в простейшем случае $(\alpha=\beta=\varepsilon=0, \gamma \neq 0)$ с помощью предложенного подхода найдены новые $(2+1)$-мерные интегрируемые уравнения гидродинамического типа.

2. При $\beta=\varepsilon=0\left(\alpha=1\right.$, константа $\gamma$ убирается сдвигом: $\left.H_{0}+\gamma \rightarrow H_{0}\right)$ аналогично получаем

$$
\Phi_{11}=\frac{\Omega_{x y}}{\Omega_{x x}}+\left(\frac{1}{\Omega_{x x}}+\frac{A}{4 \Omega_{x x}^{2}}\right) \Omega_{x t}^{2}+\left(\frac{B}{\Omega_{x x}}+\frac{C}{\Omega_{x x}^{2}}\right) z_{x t}+\frac{C^{2}}{A \Omega_{x x}^{2}}+\frac{E}{\Omega_{x x}}+G e^{A / \Omega_{x x}},
$$

где $(A, B, C, E, G)$ - произвольные константы, еще одно новое интегрируемое $(2+1)$-мерное уравнение гидродинамического типа.

3. Если положить теперь $\varepsilon=0$ ( $\beta=1, H_{0}$ и константа $\gamma$ убираются галилеевским преобразованием $\left.H_{1}+\alpha H_{0}+\gamma \rightarrow H_{1}, x-\alpha t \rightarrow x\right)$, то получаем еще одно новое $(2+1)$ интегрируемое уравнение гидродинамического типа

$$
\Omega_{t t}=\frac{\Omega_{x y}+B \Omega_{x x}+C}{\Omega_{x t}}+\frac{1}{6} w\left(\Omega_{x x}\right) \Omega_{x t}^{2},
$$

где функция $w$ является решением уравнения Шази

$$
w^{\prime \prime \prime}+2 w w^{\prime \prime}=3 w^{\prime 2}
$$

Общий случай $(\varepsilon \neq 0)$, так же как и соответствующие гидродинамические цепочки за недостатком места будут рассмотрены в отдельной публикации.

\section{СПИСОК ЛИТЕРАТУРЫ}

[1] С. П. Царев // Докл. АН СССР. 1985. Т. 282. № 3. С. 534-537. [2] М. В. Павлов, С. П. Царев // Функц. анализ и его прил. 2003. Т. 37. № 1. С. 38-54. [3] Lei Yu // J. Phys. A. 2000. V. 33. № 45. P. 8127-8138. 